\title{
Platelet-rich plasma treatment improves outcomes for chronic proximal hamstring injuries in an athletic population
}

\author{
Ryan R. Fader ${ }^{1}$ \\ Justin J. Mitchell ${ }^{1}$ \\ Shaun Traub2 \\ Roger Nichols ${ }^{2}$ \\ Michelle Roper ${ }^{1}$ \\ Omer Mei Dan ${ }^{1}$ \\ Eric C. McCarty ${ }^{1}$
}

1 University of Colorado Hospital Department of Orthopedic Surgery, Division of Sports Medicine and Shoulder Surgery, Aurora, CO, USA

2 Boulder Community Hospital, Department of Musculoskeletal Radiology, Boulder, CO, USA

Corresponding author:

Eric C. McCarty

CU Sports Medicine

University of Colorado Hospital

Department of Orthopaedic Surgery

311 Mapleton Ave.Boulder, CO 80304

E-mail: Eric.McCarty@ucdenver.edu

\section{Summary}

Background: chronic proximal hamstring tendinopathies is a disabling activity related condition. Currently, there is no well-accepted or extensively documented non-operative treatment option that provides consistently successful results.

Purpose: to evaluate the efficacy of ultrasound guided platelet-rich plasma injections in treating chronic proximal hamstring tendinopathies.

Methods: a total of 18 consecutive patients were retrospectively analyzed. All patients received a single injection of platelet rich plasma via ultrasound guidance by a single radiologist. Outcome measures included a questionnaire evaluating previous treatments, visual analog scale (VAS) for pain, subjective improvement, history of injury, and return to activity.

Results: the patient population included $12 \mathrm{fe}-$ males and 6 males. The average age at the time of the injection was 42.6 years (19-60). Provocative activities included running, biking, swimming. The average body mass index of patients was 22.9 (17.2-30.2). The average time of chronic pain prior to receiving the first injection was 32.6 months (6-120). All patients had attempted other forms of non-surgical treatment prior to entering the study. The average VAS pre-injection was 4.6 $(0-8)$. Six months after the injection, $10 / 18$ patients had $\mathbf{8 0} \%$ or greater improvement in their VAS. Overall, the average improvement was $63 \%$ (5-100). The only documented side effect was post-injection discomfort that resolved within seventy-two hours.

Conclusion: chronic hamstring tendinopathy is a debilitating condition secondary to the pain, which limits an athlete's ability to perform. For refractory cases of chronic insertional proximal hamstring injuries, platelet-rich plasma injections are safe and show benefit in the majority of patients in our study, allowing return to pre-injury activities.

Study Design: Case series; Level of evidence, 4.

KEY WORDS: proximal hamstring tendinitis, proximal hamstring tendinopathy, platelet rich plasma, overuse injury, outcome.

\section{Introduction}

Tendinopathy is a painful pathologic process within the tendon resulting in histopathological changes as well as a reduced functional capacity. Histopathologic findings include degeneration, ischemic changes, disorganized intra-tendinous structure, and a failed healing response ${ }^{1-3}$. These changes have been suggested to be the result of both intrinsic and extrinsic factors $^{3-6}$. Extrinsic factors include repetitive stress, physiological overload beyond the tendons threshold during physical training including eccentric contracture, non-uniform stress, poor training technique, and chemical stresses such as steroids or flouroquinolones ${ }^{3,6,7}$. Intrinsic factors include advanced age or physical deconditioning, genetic predisposition, or metabolic disease $e^{2,3,6,8-11}$.

The hamstring muscle complex is commonly strained and injured in athletes performing rapid, eccentric contracture movements such as acceleration or deceleration. Traditionally, this includes activities such as long distance running, sprinting, and jumping ${ }^{12,13}$. The majority of hamstring injuries occurs at the myotendinous junction, however up to $12 \%$ of injuries have been found to occur at the proximal tendinous origin on the ischial tuberosity ${ }^{14}$. Lempainen et al. obtained biopsy specimens from the ischial hamstring origin of patients with symptomatic chronic proximal hamstring tendinitis, all of which demonstrated tendinosis and 
degenerative changes within the tendon ${ }^{1}$. In addition, it has been demonstrated that these chronic proximal hamstring injuries enter into a cycle of failed reparation and re-injury, which can lead to painful, activitylimiting, chronic tendinopathy ${ }^{1}$. Furthermore, tendinous injuries occurring at the enthesis or the osteotendinous junction have a very sparse blood supply, further impeding the healing process ${ }^{7,15}$.

Despite the understanding of the underlying pathology, reported management strategies for proximal hamstring tendinosis continue to vary widely throughout the published literature $1,3,12,16,17$. Similar to other insertional tendinopathies, the initial treatment strategy in most cases is conservative, consisting of rest, activity modification, NSAIDs, or physiotherapy 3,5 . To date, the largest systematic review on the role of eccentric exercises on various sports related tendinopathies, including hamstring tendinitis indicated that eccentric exercises in hamstring tendinitis may indeed be an effective treatment modality, but more randomized controlled trials need to be completed ${ }^{18}$.

More invasive treatment options consist of injections (sclerosing agents, autologous blood products such as PRP, steroids, and anesthetics) and/or extra-corporeal shockwave therapy ${ }^{3}$. A recently published study by Cacchio et al. described shockwave therapy as a viable methodology to treat proximal hamstring tendinitis, and reported $80 \%$ improvement in symptoms ${ }^{16}$. This study, though promising, is limited by a short-term follow-up of only 3 months.

Surgical intervention, consisting of open or endoscopic debridement, ischial drilling, and sciatic release, for the treatment of chronic proximal hamstring tendinopathy has demonstrated excellent results in $60 \%$ of cases in one study ${ }^{1}$. However, there is a $10 \%$ complication rate with surgical intervention, including: paraesthesias, deep venous thromboembolism, poor wound healing, and recurrent pain requiring revision surgery ${ }^{1}$. As such, there has not yet been a standard of care treatment regimen described that is cost effective, provides minimal complication risk, and yields high treatment success rates.

There has been recent popularization and increased use of platelet rich plasma (PRP), with various reports of its benefit in treating soft tissue injuries such as tendinosis ${ }^{3,19,20}$. Despite this increasing use in soft tissue injuries, its efficacy remains controversial. It has been previously established that platelets provide regenerative potential by the process of chemotaxis ${ }^{21-25}$. This serves to promote an increase in local regenerative cell accumulation and healing via multiple processes including angiogenesis, inflammation, stimulation of precursor cells, and restoration of collagen orientation 26,27 . This is evidenced and reinforced by release of inflammatory mediators such as COX-1 and2, PGE-2 ${ }^{23,24}$. In the setting of tendinosis, where healing potential is limited and cell orientation is disorganized, this chemotaxic process would appear to be advantageous to promote tendon healing.

In an attempt to provide a cost effective, low morbidity approach to the treatment of this complex problem, we have retrospectively reviewed questionnaire-based outcomes of patients suffering from chronic proximal hamstring tendinopathy, all whom were treated with ultrasound guided platelet rich plasma (PRP) injection into the proximal hamstring origin. The objective of this study was to evaluate the efficacy of this procedure and its effect on functional outcome in the treatment of chronic proximal hamstring tendinosis.

\section{Materials and methods}

After institutional review board and ethics board approval, a prospective cohort of athletes with diagnoses of chronic proximal hamstring tendinitis were identified in the Sports Medicine Department at the University of Colorado Hospital. The study was completed in accordance with international standards set forth by Padulo et al. ${ }^{28}$. Diagnoses were identified in the clinical setting spanning an eighteen-month collection period between 2011 and 2013. Inclusion criteria were a clinical diagnosis of chronic proximal hamstring tendinopathy by a sports medicine trained orthopaedic surgeon, and confirmed by magnetic resonance imaging (MRI). In addition, the patient also had to have failed a trial of conservative management, including at least six months of activity modification or limitation in physical activity, goal-directed physical therapy, and anti-inflammatory medication. Patients with complete proximal hamstring ruptures or ischial tuberosity avulsion fractures were excluded. Patients meeting inclusion criteria were offered continued conservative management, or an ultrasound guided injection of PRP into the proximal hamstring origin. Eighteen patients meeting criteria desired PRP injection and were enrolled after informed consent was obtained. These patients filled out a pre-injection questionnaire detailing a history of the injury, previous treatments prescribed and attempted, as well as their pain score on the Visual Analog Pain Scale (VAS) from 0-10.

The Angel Whole Blood Separation System (Cytomedix Inc., Gaithersburg, MD) was used for separation of the leukocyte reduced PRP product. All injections were performed by a single fellowship trained radiologist under ultrasound guidance with use of a 22-gauge needle (Fig. 1). Over the study period, various ultrasound machines were used, as an upgrade of ultrasonography equipment was undertaken at the host institution. The Philips iU22 machine (Philips Healthcare, Andover, MA) was the most commonly and recently used machine. Local anesthetic (2-5 cc of $1 \%$ lidocaine with a $10 \%$ bicarbonate dilution) was used immediately prior to injection of platelet rich plasma (2.5-4 cc).

Post injection, patients were restricted from anti-inflammatories for 6 weeks. Activity was limited to no sporting activity and nothing more strenuous than daily at home chores for one week. Initiation of jogging without speed drills or hill/incline work was allowed at three weeks. Thereafter, patients were allowed to return to activity as desired on an individualized and as tolerated basis. 


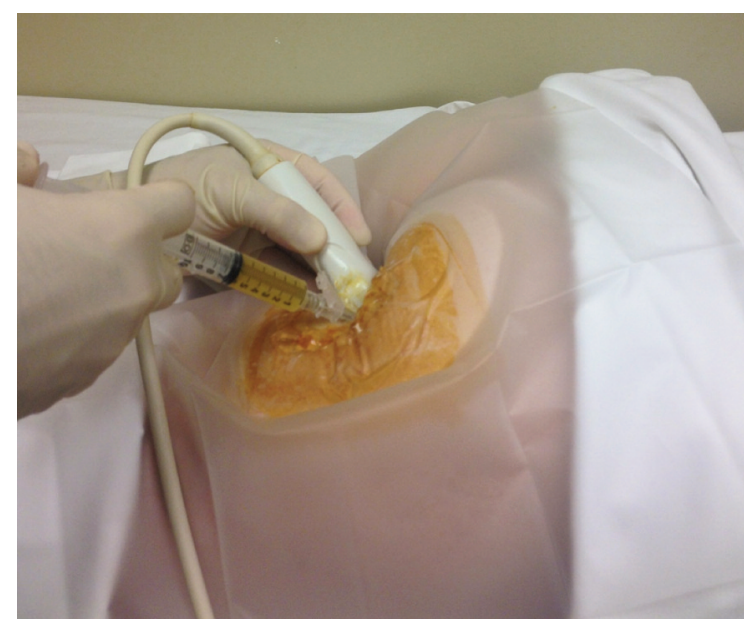

Figure 1. Bedside platelet-rich plasma injection of the proximal hamstring under ultrasound guidance.

Follow-up data was collected in a retrospective fashion on each of the eighteen patients. Each patient was followed individually for a minimum of six months. At 4-6 weeks post-injection, pain was evaluated with VAS for each subject, and they were provided a questionnaire. The questionnaire detailed whether they had pain at rest, pain with activity, whether they had been able to increase their activity level from pre-injection baseline, and what subjective percentage of improvement they had noticed. Similarly, at 3 months post-injection, they were again assessed with VAS, and another subjective questionnaire was provided, again outlining the above questions. Finally, at the 6-month-follow up they were again assessed with VAS, whether they had any pain at all, if they had returned to pre-injection activities, and their percentage of improvement.

\section{Results}

All 18 patients enrolled for PRP injection were available for follow up via the questionnaire at 6 months. Our patient population included 12 females and 6 males. The average age at the time of the injection was 42.6 years (19-60). Pre-injection activities included running, biking, swimming. There were 11 patients with left-sided hamstring pathology, and 7 patients with right-sided pathology. The average body mass index of patients was 22.9 (17.2-30.2). The average length of time the patients had chronic pain prior to receiving the first injection was 32.6 months $(6-120)$. Activities leading to injury are delineated in Table 1, and conservative treatments attempted are shown in Table 2. The average pre-injection pain score was 4.6 (range 0-8) on the Visual Analog Scale (VAS). Six months after the injection, $10 / 18$ (55.6\%) patients had an $80 \%$ or greater improvement in their subjectively reported pain. Overall, the average improvement was $63 \%(5-100)$. The only side effect experienced was post-injection discomfort that resolved within 72
Table 1. Overview of patient activity and primary sport activity. 8 patients reported running as the cause of the proximal hamstring injury, 3 patients were injured while cycling, and 2 patients were injured after a fall during recreational sports. 1 patient was injured walking and 1 patient was injured lifting weights.

\begin{tabular}{ll}
\hline Causal Activity & \# of Patients \\
\hline Running & 11 \\
Cycling & 3 \\
Fall during sport & 2 \\
Walking & 1 \\
Weight Lifting & 1
\end{tabular}

Table 2. 8 patients had failed physical therapy, 7 patients had a cortisone injection at the ischial tuberosity, 4 patients had received acupuncture, 3 patients had tried massage therapy, 2 patients had been treated by a Chiropractor, and 1 patient had prolotherapy.

\begin{tabular}{ll}
\hline Failed Treatment Modality & \# of Patients \\
\hline Cortisone Injection & 7 \\
Physical Therapy & 8 \\
Acupuncture & 4 \\
Massage Therapy & 3 \\
Chiropractor & 2 \\
Prolotherapy & 1 \\
\hline
\end{tabular}

hours with conservative measures including icing, anti-inflammatories, or watchful waiting.

\section{Discussion}

Chronic hamstring tendinitis is a well-described entity affecting athletic performance and return to play. Most chronic hamstring injuries result from tendinosis at the muscle origin site, the ischial tuberosity. This is supported by Lempainen et al., who used biopsy specimens to histologically demonstrate the presence of tendinosis at the origin of the hamstring tendons in patients with proximal hamstring tendinitis ${ }^{1}$. Management strategies, which widely vary, have been described throughout the literature. These treatment modalities include rest, physical therapy, corticosteroid injections or surgical debridement for refractory cases as described by Puranen and Orava in $1988^{29}$. Reported outcomes have been variable in success, thereby leading to difficulty in successfully and consistently treating this disorder.

Platelet rich plasma, with a platelet concentration of at least $1,000,000$ platelets/uL in $5 \mathrm{~mL}$ of plasma, is associated with the enhancement of healing due to its bioactive factors. These factors are released from the $\alpha$-granules of a platelet, and include transforming growth factor- $\beta$ (TGF- $\beta$ ), platelet derived growth factor (PDGF), insulin like growth factor 1 and 2 (ILGF 1 and 2), fibroblast growth factor (FGF), epidermal growth factor (EGF), vascular endothelial growth factor (VEGF) and endothelial cell growth factor 
$(\mathrm{ECGF})^{3,9,20}$. These factors are found to be 3 to 5 times more abundant in platelet rich plasma than in that of regular plasma, allowing for potential enhancement of healing by the delivery of these various growth factors and cytokines, as they play important roles in cell proliferation, chemotaxis, cell differentiation and angiogenesis 2,3,10,21.

Platelet rich plasma has been shown to have positive effects on tendon healing. A recent study by de Mos et al. demonstrated that platelet rich plasma positively affected gene expression and matrix synthesis in human tendons and tenocytes, leading to increasing collagen production and total collagen ${ }^{30}$. Another study by Kajikawa et al. demonstrated that platelet rich plasma injected into an injuried patellar tendon of chimeric rats expressing green fluorescent protein in circulating cells and bone marrow cells resulted in increased recruitment of circulation derived cells to the tendon injury site, with concomitant increased collagen production ${ }^{31}$.

Platelet rich plasma is increasingly being used to treat various conditions within orthopaedics, including intraarticular pathology, as well as tendinopathies. The majority of the current available reports on this are level 5 studies, many of which demonstrate improved healing and reduced pain when using PRP to treat various tendinopathies, including the achilles tendon, patellar tendon, supraspinatous tendon, and the mobile wad in lateral epicondylitis. A recent systematic review delineated the science behind PRP, and suggested a therapeutic effect when used intra-articularly ${ }^{32}$. Kon et al. demonstrated a statistically significant improvement in 20 patients with chronic patellar tendinitis at 6 months who underwent a series of three PRP injections ${ }^{33}$. Similarly, Filardo et al. performed a randomized controlled trial of 31 patients with chronic patellar tendinitis. Of these, 15 were treated with a series of three PRP injections two weeks apart, while 16 were treated with $\mathrm{PT}$ alone. They demonstrated an improvement in pain, EQ VAS, and Tegner scores at 6 months in those patients treated with PRP relative to those treated with PT alone ${ }^{34}$. More recently, a large systematic review and network meta-analysis of randomized controlled trials evaluated various injection therapies for the treatment of lateral epicondylitis, which demonstrated a statistically significant superiority of PRP to placebo ${ }^{35}$.

Specific to our indication, Wetzel et al. presented a retrospective cohort of 15 patients who underwent PRP injection for chronic proximal hamstring tendinitis after failed conservative management ${ }^{36}$. Their study, the first of its kind, demonstrated that all study subjects who failed conservative measures and subsequently underwent injection to the hamstring origin experienced significant reduction in subjective VAS pain scores (average 7.2 pre-injection versus 1.2 post-injection), and that all patients (including 9 high level athletes) returned to their previous level of activity, with only one requiring a second injection to do so. Other than the afore mentioned repeat injection, there were no noted complications with this treatment. This study, though promising with regard to treatment modality, has not been verified and had a relatively small number of subjects. Through the current study we sought to help verify these preliminary results, and improve indications for this new, less invasive treatment modality, which can potentially provide good to excellent symptom relief through Platelet Rich Plasma ultrasound guided injections at the hamstrings origin.

The technique of PRP injection has been criticized, as it can vary widely. Variables include leukocyte rich versus leukocyte poor PRP, activated versus non-activated PRP, and variance in use of imaging for needle placement. Current knowledge regarding the former two variables is overall lacking in the literature. Dragoo et al. compared leukocyte reduced and leukocyte rich PRP injections in rabbit tendons ${ }^{25}$. They found an increase in the cellular immune response with in the tendon at 5 days after the injection; however there was no difference in the intra-tendinous immune response by 14 days post injection ${ }^{25}$. The use of imaging has been demonstrated to significantly increase the accuracy of needle placement ${ }^{37,38}$. Amongst various modalities, ultrasound has been demonstrated to be one of the most accurate modalities for injections ${ }^{37,38}$. In addition, it is both accessible and allows for direct identification of pathology. Finnoff et al. compared fluoroscopy to ultrasound guidance for piriformis injections in 20 cadaver extremities. In the group injected with use of ultrasound, $19 / 20(95 \%)$ demonstrated liquid latex within the piriformis, compared with only $6 / 20(30 \%)$ of the fluoroscopic guided contrast-controlled group $(p=0.001)^{37}$. Ultrasound guidance for injections has also been confirmed to increase the accuracy of injection in the setting of intra-articular injections, where randomizedcontrolled studies demonstrated a better clinical outcome in those patients who underwent ultrasoundguided injections compared to those who did not ${ }^{39}$. Because of these prior studies, as well as our recent experience, we feel our current technique is both valid and is likely to produce the greatest percentage of accurate injections over time when compared to blind or fluoroscopically guided injections.

The results of our study indicate that ultrasound guided platelet rich plasma injection at the ischial tuberosity for chronic recalcitrant hamstring tendinitis is an effective reliable treatment modality when traditional conservative treatment fails. Some cases can certainly be treated conservatively and achieve successful results, but other cases do not respond as well to these modalities, and the literature suggests either a corticosteroid injection or operative repair as the next possible step ${ }^{35}$. Our results support the prior results by Wetzel et al. that PRP would be a logical, and less invasive, option for treating proximal hamstrings tendinopathy, specifically those that have failed traditional conservative treatment. Platelet rich plasma injection provides improvement in functional outcomes, while providing pain relief. Side effects included postinjection discomfort in one of the eighteen patients $(0.056 \%)$, which spontaneously resolved. This was classified as a minor complication. Due to the suc- 
cess of the platelet rich plasma injections to relieve symptoms in chronic hamstring tendinitis that was refractory to other non-operative management strategies, we believe this is a viable and excellent treatment concept for this patient population.

\section{Limitations}

There are clear limitations to this study. Our cohort is small and from a generally active and fit population, possibly making broad generalization difficult. Further, this study did not examine a control group, but rather individuals acted as their own internal control, having undergone non-invasive treatment prior to the PRP injection. A larger, randomized controlled trial with standardized outcome measures and long-term follow-up is needed to completely assess the validity of PRP as a treatment option, as well as the optimal regimen.

\section{References}

1. Lempainen L, Sarimo J, Mattila K, Vaittinen S, Orava S. Proximal hamstring tendinopathy results of surgical management and histopathologic findings. Am J Sports Med. 2009;37(4): 727-734.

2. Abate M, Silbernagel KG, Siljeholm C, et al. Pathogenesis of tendinopathies: inflammation or degeneration? Arthritis Res Ther. 2009;11:235.

3. Andia I, Latorre PM, Gomez MC, Burgos-Alonso N, Abate M, Maffulli N. Platelet-rich plasma in the conservative treatment of painful tendinopathy: a systematic review and meta-analysis of controlled studies. Br Med Bull. 2014;110(1): 99-115.

4. Williams JG. Achilles tendon lesions in sport. Sports Med. 1986;3:114-135

5. Valent A, Frizziero A, Bressan S, Zanella E, Giannotti E, Masiero $S$. Insertional tendinopathy of the adductors and rectus abdominis in athletes: a review. Muscles Ligaments Tendons J. 2012;10(2):142-148.

6. Frizziero A, Bonsangue V, Trevisan M, Ames PR, Masiero S. Foot tendinopathies in rheumatic diseases: etiopathogenesis, clinical manifestations and therapeutic options. Clin Rheumatol. 2013;32(5):547-55.

7. Sharma P, Maffulli N. Biology of tendon injury: healing, modeling, and remodeling. J Musculoskelet Neuronal Interact. 2006;6:181-190.

8. Abate M, Oliva F, Schiavone C, Salini V. Achilles tendinopathy in amateur runners: role of adiposity (Tendinopathies and obesity). Muscles Ligaments Tendons J. 2012;2:44-48.

9. Abate M, Schiavone C, Salini V, Andia I. Occurrence of tendon pathologies in metabolic disorders. Rheumatology. 2013;52:599-608.

10. Andia I, Sánchez M, Maffulli N. Tendon healing and platelet rich plasma therapies. Expert Opin BiolTher. 2010;10:14151426.

11. Andia I, Maffulli N. Platelet rich plasma for managing pain and inflammation in osteoarthritis. Nature Review Rheum. 2013;9: 721-730.

12. Ahmad CS, Redler LH, Ciccotti MG, Maffulli N, Longo UG, Bradley J. Evaluation and management of hamstring injuries. Am J Sports Med. 2013;41(12):2933-2947.

13. Brubaker CE, James SL. Injuries to runners. J Sports Med. 1974;2(4):189-198.

14. Koulouris G, Connell D. Evaluation of the hamstring muscle complex following acute injury. Skeletal Radiol. 2003;32:582589.

15. Kannus $P$, Jozsa $L$, Jarvinnen $M$. Basic science of tendons. In: Garrett Jr, WE, Speer KP, Kirkendall DT eds Principles and Practice of Orthopaedic Sports Medicine. Philadelphia; Lippincott Williams \&Wilkins. 2000;21-37.

16. Cacchio A, Rompe JD, Furia JP, Santilli V, De Paulis F. Shockwave therapy for the treatment of chronic proximal hamstring tendinopathy in professional athletes. Am J Sports Med. 2011;39(1):146-153.

17. Clanton T, Coupe K. Hamstring strains in athletes: diagnosis and treatment. J Am Acad Orthop Surg. 1998;6(4):237-248.

18. Frizziero A, Trainito S, Oliva F, Aldini N, Masiero S, Maffulli N The role of eccentric exercise in sport injuries rehabilitation. $\mathrm{Br}$ Med Bulletin. 2014;110(1):47-75.

19. Mei-Dan O, Carmont MR. The role of platelet-rich plasma in rotator cuff repair. Sports Med Arthrosc. 2011;19(3):244-250.

20. Mei-Dan O, Lippi G, Sanchez M, Andia I, Maffulli N. Autologous platelet-rich plasma: a revolution in soft tissue sports injury management? Phys Sportsmed. 2010;4(38):127-135.

21. Engebretson L, Steffen K, Alsousou J, et al. IOC consensus paper on the use of platelet-rich plasma in sports medicine. $\mathrm{Br}$ J Sports Med. 2010;44:1072-1081.

22. Yeaman MR. The role of platelets in antimicrobial hos defense. Clin Infect Dis. 1997;25:951-968.

23. Fredberg U, Stengaard-Pedersen K. Chronic tendinopathy tissue pathology, pain mechanisms, and etiology with a special focus on inflammation. Scand J Med Sci Sports. 2008;18:3-15.

24. Zhang J, Middleton KK, Fu FH, Im HJ, Wang JH. HGF mediates the anti-inflammatory effects of PRP on injured tendons. PLoS One. 2013;8(6):1-12.

25. Dragoo JL, Braun HJ, Durham JL, et al. Comparison of the acute inflammatory response of two commercial platelet-rich plasma systems in healthy rabbit tendons. Am J Sports Med. 2012;40(6):1274-1281.

26. Anitua E, Sanchez M, Nurden AT, et al. Reciprocal actions of platelet-secreted TGF-beta1 on the production of VEGF and HGF by human tendon cells. Plast Reconstr Surg 2007;119: 950-959.

27. Anitua E, Sanchez M, Nurden AT, et al. Autologous fibrin matrices: a potential source of biological mediators that modulate tendon cell activities. J Biomed Mater Res A. 2006;77:285293.

28. Padulo J, Oliva F, Frizziero A, Maffulli N. Muscles, Ligaments and Tendons Journal. Basic principles and recommendations in clinical and field science research. 2013;4:250-252.

29. Puranen J, Orava S. The hamstring syndrome: a new diagnosis of gluteal sciatic pain. Am J Sports Med. 1988;16:517-521.

30. deMos $M$, vad der Windt $A E$, Jahr $H$, et al. Can platelet-rich plasma enhance tendon repair? A cell culture study. Am JSports Med. 2008;36(6):1171-1178.

31. Kajikawa Y, Morihara T, Sakamoto H, et al. Platelet-rich plasma enhances the initial mobilization of circulation-derived cells for tendon healing. J Cell Phys. 2008;215(3):837-845.

32. Frizziero A, Giannotti E, Ferraro C, Masiero S. Platelet rich plasma intra-articular injections: A new therapeutic strategy for the Treatment of knee osteoarthritis in sport rehabilitation. A systematic review. Sport Sciences for Health. 2012;8(1):15-22.

33. Kon E, Filardo G, Delcogliano M, et al. Platelet-rich plasma: New clinical application: A pilot study for treatment of jumper's knee. Injury. 2009;40(6):598-603.

34. Filardo G, Kon E, Della Villa S, Vincentelli F, Fornasari PM, Maracci M. Use of platelet-rich plasma for the treatment of refractory jumper's knee. Int Orthop. 2010;34(6):909-915.

35. Krogh TP, Bartels EM, Ellingsen T, et al. Comparative effectiveness of injection therapies in lateral epicondylitis: a systematic review and network meta-analysis of randomized controlled trials. Am J Sports Med. 2013;41(6):1435-1446. 
36. Wetzel RJ, Patel RM, Terry MA. Platelet-rich plasma as an effective treatment for proximal hamstring injuries. Orthopedics. 2013;36(1):e64-70.

37. Finnoff JT, Hurdle MF, Smith J. Accuracy of ultrasound-guided versus fluoroscopically guided contrast-controlled piriformis injections. J Ultrasound Med. 2008;27:1157-1163.

38. Zissen MH, Wallace G, Stevens KJ, Fredericson M, Beaulieu
CF. High hamstring tendinopathy: MRI and ultrasound imaging and therapeutic efficacy of percutaneous corticosteroid injection. Am J of Roentgenology. 2010;195(4):993-998.

39. Soh E, Li W, Ong KO, Chen W, Bautista D. Image-guided versus blind corticosteroid injections in adults with shoulder pain: a systematic review. BMC Musculoskelet Disord. 2011; 12:137. 